\title{
Associations between TGF- $\beta 1$ Levels and Markers of Hemolysis, Inflammation, and Tissue Remodeling in Pediatric Sickle Cell Patients
}

\author{
Rayra P. Santiago $\mathbb{D}^{1,2}$ Magda O. S. Carvalho, ${ }^{3}$ Camylla V. B. Figueiredo $\mathbb{D},{ }^{1,2}$ \\ Luciana M. Fiuza $\mathbb{D}^{\mathbb{1}}{ }^{1,2}$ Rodrigo M. Oliveira $\mathbb{D}^{1}{ }^{1,2}$ Sètondji C. M. A. Yahouédéhou $\left(\mathbb{D},{ }^{1,2}\right.$ \\ Valma M. L. Nascimento, ${ }^{4}$ Isa M. Lyra, ${ }^{3,4}$ Théo Araujo-Santos $\mathbb{D},{ }^{1}$ Nívea F. Luz $\mathbb{D}$, ${ }^{1}$ \\ Milena M. Aleluia $\left(\mathbb{D},{ }^{5}\right.$ Caroline C. Guarda $\mathbb{D}^{1,2}{ }^{1,2}$ Valéria M. Borges $\mathbb{D}^{1}{ }^{1}$ \\ and Marilda S. Goncalves $\mathbb{D}^{1,2}$ \\ ${ }^{1}$ Instituto Gonçalo Moniz, Fundação Oswaldo Cruz, 40.296-710 Salvador, Brazil \\ ${ }^{2}$ Universidade Federal da Bahia, 40.170-110 Salvador, Brazil \\ ${ }^{3}$ Hospital Universitário Professor Edgard Santos, Universidade Federal da Bahia, 40110-060 Salvador, Brazil \\ ${ }^{4}$ Fundação de Hematologia e Hemoterapia do Estado da Bahia, 40286-240 Salvador, Brazil \\ ${ }^{5}$ Departamento de Ciências Biológicas, Universidade Estadual de Santa Cruz, 45.662-900 Ilhéus, Brazil
}

Correspondence should be addressed to Marilda S. Goncalves; mari@bahia.fiocruz.br

Received 9 June 2020; Accepted 1 March 2021; Published 15 March 2021

Academic Editor: Daniela Caccamo

Copyright (c) 2021 Rayra P. Santiago et al. This is an open access article distributed under the Creative Commons Attribution License, which permits unrestricted use, distribution, and reproduction in any medium, provided the original work is properly cited.

\begin{abstract}
Transforming growth factor beta (TGF- $\beta$ ) is a cytokine with important involvement in biological processes related to the pathogenesis of sickle cell disease (SCD), including endothelial and vascular dysfunction, inflammation, and hematopoietic homeostasis. This study is aimed at investigating associations between levels of TGF- $\beta 1$ and classical laboratory biomarkers and inflammatory mediators, as well as the tissue inhibitor of metalloproteases-1 (TIMP-1) and matrix metalloproteinase-9 (MMP-9), in pediatric patients $(n=123)$ with SCD in steady state: 84 with sickle cell anemia (HbSS) and 39 with hemoglobin SC disease (HbSC). A healthy control (HC) group of 59 individuals was also included. Hematological and biochemical analyses were carried out using electronic methods. TGF- $\beta 1$, TIMP-1, and MMP-9 plasma quantifications were performed by ELISA. TGF- $\beta 1$ plasma levels were higher in HbSS individuals than in HbSC and HC. In individuals with HbSS, TGF- $\beta 1$ levels were positively correlated with red blood cells, hemoglobin, hematocrit, platelets, and TIMP-1. In addition, HbSS individuals with TGF- $\beta 1$ levels above the median $(\geq 72.29 \mathrm{ng} / \mathrm{mL})$ also presented increased monocyte counts and decreased albumin levels. In patients with HbSC, TGF- $\beta 1$ levels were positively correlated with leukocytes, eosinophils, lymphocytes, monocytes, and platelets, as well as levels of TIMP-1, VLDL-C, triglycerides, heme, and AST. Additionally, HbSC individuals with TGF- $\beta 1$ levels above the median $(\geq 47.80 \mathrm{ng} / \mathrm{mL})$ presented increased leukocyte and platelet counts, as well as increased levels of triglycerides, VLDL-C, MMP-9, and TIMP-1, and decreased HDL-C. Our findings suggest that TGF- $\beta 1$ may play important roles in vascular remodeling, vasculopathy, angiogenesis, and inflammation in pediatric patients with $\mathrm{SCD}$.
\end{abstract}

\section{Introduction}

Sickle cell disease (SCD) is a group of disorders characterized by the presence of the hemoglobin variant $S(\mathrm{HbS})$. Sickle cell anemia (SCA), the most severe type of SCD, represents homozygosity in the inheritance of the beta allele S (HbSS). Hemoglobin SC disease (HbSC) is characterized by the association of $\mathrm{HbS}$ with another hemoglobin variant, hemoglobin $\mathrm{C}(\mathrm{HbC})$, and is considered a milder phenotype of SCD [1-4]. 
Individuals with SCD exhibit an acute and chronic inflammatory status associated with recurrent infections and increased leukocyte counts, as well as the activation of leukocytes, red blood cells, reticulocytes, and endothelial cells. These individuals often experience clinical events related to vascular dysfunction, such as priapism, pulmonary hypertension, vasoocclusive crisis (VOC), and stroke $[5,6]$.

Vascular dysfunction is a common feature in SCD, mainly caused by nitric oxide (NO) imbalance in association with chronic hemolysis [5, 7]. During intravascular hemolysis, free hemoglobin and heme react with $\mathrm{NO}$, causing the degradation of this free radical. The hemolytic process also induces the release of arginase, which consumes arginine, the main substrate for $\mathrm{NO}$ production. These events result in reactive oxygen and nitrogen species (ROS and RNS) production, which contribute to cell damage and vascular dysfunction by way of nitrosative stress [6].

The chronic inflammatory status observed in SCD has also been linked to increased levels of inflammatory cytokines, such as interleukin- (IL-) 1, tumor necrosis factor-alpha (TNF- $\alpha$ ), and endothelin-1, which are produced by activated endothelial cells [8]. SCA patients have been described to present increased plasma levels of TNF- $\alpha$, IL-8, and prostaglandin $\mathrm{E}_{2}\left(\mathrm{PGE}_{2}\right)$ [9]. In addition, transforming growth factor beta 1 (TGF- $\beta 1$ ), IL17 , and IL-18 are capable of activating the vascular endothelium $[8,10]$. In SCD, patterns of cytokine production vary during steady and crisis state [11], suggesting that the inflammatory response is capable of modulating clinical events.

TGF- $\beta$ is a pleiotropic family of cytokines produced by many cell types, such as immune cells ( $\mathrm{T}$ cells and macrophages), tumor cells, and stromal cells. These growth factors have been implicated in the regulation of cell growth, proliferation, differentiation, adhesion, and migration, as well as Th17 response polarization, ROS production, and apoptosis [12-16]. In the context of SCD, TGF- $\beta$ is involved in several processes, including wound healing/ulceration, proliferative vasculopathy, inflammation, immune response, and ROS production $[10,17]$, all of which have been linked to clinical events.

TGF- $\beta$ can upregulate the expression and activity of matrix metalloproteinases 9 (MMP-9), as well as its antagonist, tissue inhibitor of metalloproteases-1 (TIMP-1) [18, 19]. MMP-9, a zinc-dependent endopeptidase that acts in the extracellular matrix, promotes tissue remodeling in response to physiological and pathological conditions [1821] Additionally, MMP-9 can also activate cytokine and chemokine production and is a regulator of inflammation and immunity [10]. Interplay between TIMP-1 and MMP-9 is involved in angiogenesis, cell growth, and apoptosis [22, 23].

Considering the immunomodulatory role of TGF- $\beta$ together with the plethora of effects exerted by this superfamily, we hypothesized that TGF- $\beta 1$ may play a key role in the pathogenesis of SCD. Hence, we sought to investigate associations between plasma levels of TGF- $\beta 1$ and classical laboratory biomarkers, as well as TIMP-1 and MMP-9, in individuals with HbSS and HbSC.

\section{Material and Methods}

2.1. Subjects. Pediatric patients with SCD $(n=123)$ were recruited from the Bahia State Hematology and Hemother- apy Foundation (HEMOBA), located in Salvador, Brazil. Of these, 84 were HbSS and 39 were HbSC; 37 (44.04\%) and $19(48.71 \%)$ were female, respectively. All individuals were in steady-state, signifying the absence of acute events in the three months prior to inclusion, and none were undergoing hydroxyurea therapy. The mean age of patients with HbSS and $\mathrm{HbSC}$ was $8.76 \pm 3.78$ and $10.72 \pm 4.24$ years, respectively.

A healthy control (HC) group consisting of 59 individuals was included, 30 (50.85\%) of whom were female, with mean age of $8.38 \pm 3.50$ years. These individuals were recruited from the Laboratory of Clinical and Toxicological Analysis, College of Pharmaceutical Sciences, Federal University of Bahia (LACTFAR-UFBA).

This research protocol was approved by the institutional research board of the Gonçalo Moniz Institute (protocol number: 0016.0.225.000-09) and was conducted in accordance with the 1964 Declaration of Helsinki and its subsequent revisions. All individuals were informed regarding the purpose and procedures of this study, and informed written consent was obtained from each patient's legal guardian.

2.2. Hematological and Biochemical Parameters. Hematological parameters were quantified using a Coulter Count T-890 electronic cell counter (Coulter Corporation, Hialeah, Florida, USA). Reticulocytes were counted after staining supravitally with brilliant cresyl blue dye. Hemoglobin profiles and fetal hemoglobin (HbF) levels were determined by highperformance liquid chromatography using an HPLC/Variant-II hemoglobin testing system (Bio-Rad, Hercules, California, USA).

Biochemical parameters were measured using an automated A25 chemistry analyzer (Biosystems S.A., Barcelona, Catalunya, Spain). Serum ferritin was measured by immunoassay using an Access ${ }^{\circledR} 2$ immunoassay system (Beckman Coulter Inc., Pasadena, California, USA). In addition, alpha 1 antitrypsin (AAT) levels were determined using an IMMAGE $^{\circledR}$ Immunochemistry System (Beckman Coulter Inc., Pasadena, California, USA). Total systemic free heme was measured in plasma samples using the QuantiChrom Heme Assay Kit (BioAssay Systems, Hayward, California, USA) following the manufacturer's protocol.

Laboratory analysis was performed at the Laboratory of Genetic Investigation and Translational Hematology at the Gonçalo Moniz Institute-FIOCRUZ (LIGHT-IGM/FIOCRUZ) and at LACTFAR-UFBA.

2.3. TGF- $\beta 1$, TIMP-1, and MMP-9 Plasma Measurement. TGF- $\beta 1$, TIMP-1, and MMP-9 plasma levels were measured by ELISA (R\&D Systems, Minneapolis, Minnesota, USA) in accordance with the manufacturer's protocol.

2.4. Statistical Analysis. All analyses were performed using the Statistical Package for the Social Sciences (SPSS) software, version 20.0 (IBM, Armonk, New York, USA) and GraphPad Prism version 6.0 (GraphPad Software, San Diego, California, USA), which was also used for graph assembly. Significance was considered when $p<0.05$. Variable values were summarized as means. To perform comparisons among SCD phenotypes, subgroups of individuals were formed 
TABLE 1: Laboratory profile of pediatric patients with sickle cell anemia (HbSS), hemoglobin SC disease (HbSC), and healthy controls (HC).

\begin{tabular}{|c|c|c|c|c|}
\hline Parameter & $\begin{array}{c}\text { HbSS } \\
N=84 \\
(\text { mean } \pm \mathrm{SD})\end{array}$ & $\begin{array}{c}\text { HbSC } \\
N=39 \\
(\text { mean } \pm \mathrm{SD})\end{array}$ & $\begin{array}{c}\mathrm{HC} \\
N=59 \\
(\text { mean } \pm \mathrm{SD})\end{array}$ & $p$ value \\
\hline \multicolumn{5}{|l|}{ Hemoglobin pattern } \\
\hline Fetal hemoglobin (\%) & $9.79 \pm 6.17$ & $2.69 \pm 1.42$ & $0.63 \pm 0.51$ & $<0.0001$ \\
\hline S hemoglobin (\%) & $86.64 \pm 6.41$ & $51.17 \pm 6.31$ & - & $<0.0001^{*}$ \\
\hline \multicolumn{5}{|l|}{ Hematological parameters } \\
\hline $\mathrm{RBC}\left(\times 10^{6} / \mathrm{mL}\right)$ & $2.68 \pm 0.59$ & $4.30 \pm 0.57$ & $4.70 \pm 0.34$ & $<0.0001$ \\
\hline Hemoglobin (g/dL) & $7.83 \pm 1.30$ & $11.14 \pm 1.23$ & $12.90 \pm 0.94$ & $<0.0001$ \\
\hline Hematocrit (\%) & $24.19 \pm 4.00$ & $34.73 \pm 3.61$ & $38.79 \pm 2.59$ & $<0.0001$ \\
\hline MCV (fL) & $91.70 \pm 10.54$ & $80.67 \pm 7.80$ & $82.62 \pm 4.77$ & $<0.0001$ \\
\hline $\mathrm{MCH}(\rho \mathrm{g})$ & $29.73 \pm 3.66$ & $25.89 \pm 2.53$ & $27.48 \pm 1.79$ & $<0.0001$ \\
\hline $\mathrm{MCHC}(\%)$ & $32.39 \pm 0.97$ & $32.08 \pm 0.94$ & $33.25 \pm 0.68$ & $<0.0001$ \\
\hline Reticulocyte count (\%) & $9.25 \pm 4.63$ & $4.06 \pm 2.50$ & $0.86 \pm 0.28$ & $<0.0001$ \\
\hline Leukocyte count (/mL) & $14734.52 \pm 5090.44$ & $9802.56 \pm 5988.29$ & $7313.56 \pm 2448.04$ & $<0.0001$ \\
\hline Neutrophil count (/mL) & $5972.00 \pm 473.00$ & $4349.00 \pm 2095.00$ & $3430.15 \pm 1937.21$ & $<0.0001$ \\
\hline Eosinophil count $(/ \mathrm{mL})$ & $751.70 \pm 423.40$ & $562.00 \pm 462.20$ & $452.36 \pm 467.08$ & $<0.0001$ \\
\hline Lymphocyte count (/mL) & $5953.00 \pm 1973.00$ & $3076.00 \pm 1241.00$ & $2889.17 \pm 957.95$ & $<0.0001$ \\
\hline Monocyte count (/mL) & $882.00 \pm 311.10$ & $459.90 \pm 254.80$ & $510.16 \pm 221.06$ & $<0.0001$ \\
\hline Platelet count $\left(\times 10^{3} / \mathrm{mL}\right)$ & $439.18 \pm 128.10$ & $319.33 \pm 175.30$ & $313.73 \pm 67.59$ & $<0.0001$ \\
\hline \multicolumn{5}{|l|}{ Biochemical parameters } \\
\hline Total cholesterol (mg/dL) & $122.00 \pm 25.38$ & $119.00 \pm 25.96$ & $157.85 \pm 33.41$ & $<0.0001$ \\
\hline $\mathrm{HDL}-\mathrm{C}(\mathrm{mg} / \mathrm{dL})$ & $32.20 \pm 9.40$ & $41.62 \pm 12.62$ & $48.19 \pm 14.75$ & $<0.0001$ \\
\hline LDL-C (mg/dL) & $67.39 \pm 22.50$ & $59.77 \pm 21.03$ & $88.87 \pm 33.30$ & $<0.0001$ \\
\hline VLDL-C (mg/dL) & $22.36 \pm 10.01$ & $17.44 \pm 5.20$ & $19.60 \pm 10.34$ & 0.0180 \\
\hline Triglycerides (mg/dL) & $111.96 \pm 50.02$ & $87.87 \pm 26.16$ & $97.75 \pm 51.47$ & 0.0150 \\
\hline Total bilirubin (mg/dL) & $3.39 \pm 1.81$ & $1.75 \pm 1.05$ & $0.57 \pm 0.23$ & $<0.0001$ \\
\hline Direct bilirubin (mg/dL) & $0.81 \pm 0.51$ & $0.47 \pm 0.27$ & $0.23 \pm 0.07$ & $<0.0001$ \\
\hline Indirect bilirubin $(\mathrm{mg} / \mathrm{dL})$ & $2.58 \pm 1.66$ & $1.27 \pm 0.97$ & $0.30 \pm 0.20$ & $<0.0001$ \\
\hline $\mathrm{LDH}(\mathrm{U} / \mathrm{L})$ & $1054.20 \pm 522.09$ & $518.38 \pm 293.41$ & $420.90 \pm 87.15$ & $<0.0001$ \\
\hline $\operatorname{ALT}(\mathrm{U} / \mathrm{L})$ & $24.31 \pm 15.90$ & $20.17 \pm 11.78$ & $18.22 \pm 8.12$ & $<0.0001$ \\
\hline AST (U/L) & $56.79 \pm 26.92$ & $33.15 \pm 14.94$ & $33.78 \pm 12.16$ & $<0.0001$ \\
\hline Total protein $(\mathrm{g} / \mathrm{dL})$ & $7.31 \pm 0.99$ & $7.24 \pm 0.67$ & $7.08 \pm 0.65$ & 0.1450 \\
\hline Albumin $(g / d L)$ & $3.99 \pm 0.77$ & $4.06 \pm 0.54$ & $4.19 \pm 0.49$ & 0.577 \\
\hline Globulin (g/dL) & $3.31 \pm 0.86$ & $3.17 \pm 0.67$ & $2.89 \pm 0.55$ & 0.0080 \\
\hline Ferritin $(\eta \mathrm{g} / \mathrm{mL})$ & $265.20 \pm 209.10$ & $152.20 \pm 102.90$ & $31.16 \pm 13.57$ & $<0.0001$ \\
\hline Urea $(\mathrm{mg} / \mathrm{dL})$ & $17.55 \pm 6.73$ & $19.00 \pm 6.49$ & $21.87 \pm 6.63$ & $<0.0001$ \\
\hline Creatinine $(\mathrm{mg} / \mathrm{dL})$ & $0.43 \pm 0.18$ & $0.54 \pm 0.15$ & $0.58 \pm 0.18$ & $<0.0001$ \\
\hline Alpha-1 antitrypsin (mg/dL) & $166.92 \pm 40.75$ & $139.95 \pm 42.45$ & $148.50 \pm 44.04$ & $<0.0001$ \\
\hline
\end{tabular}

RBC: red blood cells; MCV: mean corpuscular volume; MCH: mean corpuscular hemoglobin; MCHC: mean corpuscular hemoglobin concentration; HDL-C: high-density lipoprotein cholesterol; LDL-C: low-density lipoprotein cholesterol; VLDL-C: very low-density lipoprotein cholesterol; AST: aspartate aminotransferase; ALT: alanine aminotransferase; LDH: lactate dehydrogenase; $N$ : number; SD: standard deviation. All $p$ values were obtained using the Kruskal-Wallis test, with the exception of $(*)$, which indicates a $p$ value obtained from the ordinary one-way ANOVA.

according to TGF- $\beta 1$ median values. The Shapiro-Wilk test was used to determine the distribution of quantitative variables. Depending on distribution, comparisons of two numerical variables were performed using the independent $t$-test and Mann-Whitney $U$ test. Ordinary one-way ANOVA or Kruskal-Wallis test was performed to compare three numerical variables depending on distribution. Spearman's rank correlation coefficient or Pearson's correlation 
coefficient were used to measure the strength of linear relationships between paired variables.

\section{Results}

3.1. Hematological and Biochemical Laboratory Parameters. Table 1 lists the hematological and biochemical laboratory parameters of the investigated $\mathrm{HbSS}, \mathrm{HbSC}$, and $\mathrm{HC}$ individuals. Comparisons among the hematological and biochemical parameters in these individuals revealed statistically significant differences in hemolytic and inflammatory markers, leukocyte counts, as well as biomarkers of lipid and iron metabolism, and hepatic and renal function.

In comparison to individuals with $\mathrm{HbSC}$ and $\mathrm{HC}$, patients with HbSS presented significantly decreased red blood cell (RBC) counts and hemoglobin (Hb), hematocrit (Ht), highdensity lipoprotein cholesterol (HDL-C), urea, and creatinine levels, in addition to increased mean corpuscular volume (MCV), mean corpuscular hemoglobin $(\mathrm{MCH})$, mean corpuscular hemoglobin concentration (MCHC), total bilirubin, indirect bilirubin, direct bilirubin, lactate dehydrogenase $(\mathrm{LDH})$, fetal hemoglobin $(\mathrm{HbF})$, iron, very low-density lipoprotein cholesterol (VLDL-C), triglyceride, aspartate aminotransferase (AST), alanine aminotransferase (ALT), alpha 1 antitrypsin (AAT) and ferritin levels, as well as counts of reticulocytes, leukocytes, neutrophils, eosinophils, lymphocytes, monocytes, and platelets.

3.2. HbSS Individuals Present Increased Plasma Levels of TGF- $\beta 1$. HbSS individuals presented higher plasmatic TGF$\beta 1$ levels than $\mathrm{HC}$ and HbSC individuals (SS $>\mathrm{HC}>\mathrm{SC}$ ). Individuals with $\mathrm{HbSS}$ presented mean TGF- $\beta 1$ levels of $70.80 \pm 23.11 \mathrm{ng} / \mathrm{mL}$, with median values of $72.29 \mathrm{ng} / \mathrm{mL}$ (IQR: $54.23-87.68 \mathrm{ng} / \mathrm{mL}$ ), versus HC: mean $62.63 \pm 19.44$ $\mathrm{ng} / \mathrm{mL}$ and median $61.90 \mathrm{ng} / \mathrm{mL}$ (IQR: $49.10-73.60 \mathrm{ng} / \mathrm{mL}$ ). Individuals with $\mathrm{HbSC}$ presented mean TGF- $\beta 1$ levels of $51.43 \pm 23.76 \mathrm{ng} / \mathrm{mL}$ and a median value of $47.80 \mathrm{ng} / \mathrm{mL}$ (IQR: $38.24-67.51 \mathrm{ng} / \mathrm{mL}$ ) (Figure 1).

3.3. Correlations between TGF- $\beta 1$ Plasma Levels and Laboratory Parameters. In individuals with HbSS, TGF- $\beta 1$ was positively correlated with RBC $(r=0.282 ; p=0.0094)$, $\mathrm{Hb}(r=0.254 ; p=0.0197)$, Ht $(r=0.284 ; p<0.0089)$, platelets $(r=0.663 ; p<0.0001)$, and TIMP-1 $(r=0.381 ; p=0.0005)$ (Figure 2(a)). In addition, in HbSC individuals, TGF- $\beta 1$ was positively correlated with leukocytes $(r=0.5168 ; p=0.0008)$, eosinophils $(r=0.3619 ; p=0.0236)$, lymphocytes $(r=0.6575$; $p<0.0001)$, monocytes $(r=0.4421 ; p=0.0048)$, platelets $(r=0.5318 ; p=0.0005)$, AST $(r=0.326 ; p=0.0425)$, VLDL$\mathrm{C}(r=0.424 ; p=0.0072)$, triglycerides $(r=0.439 ; p=0.0051)$, heme $(r=0.426 ; p=0.0076)$, and TIMP-1 $(r=0.408 ; p=$ 0.0110 ) (Figure $2(\mathrm{~b})$ ).

3.4. TGF- $\beta 1$ Plasma Levels Are Associated with Laboratory Parameters in SCD. Considering the fact that no standard or normal clinical range exists with respect to plasma levels of TGF- $\beta 1$ in humans, we endeavored to perform association analyses by creating subgroups of individuals with HbSS and HbSC according to the median values of TGF- $\beta 1$ obtained for each SCD genotype.

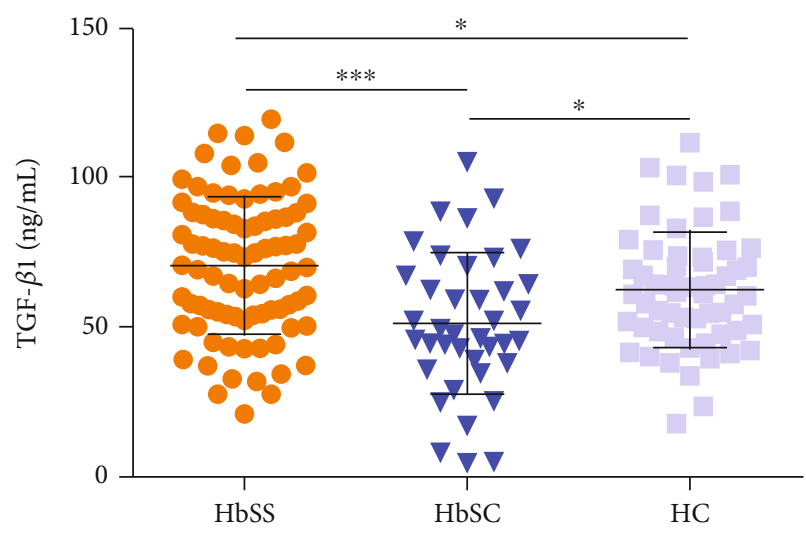

Figure 1: TGF- $\beta 1$ plasmatic levels in individuals with HbSS, HbSC, and healthy controls (HC). ${ }^{*} p<0.05 ;{ }^{* * *} p<0.0001$.

In individuals with HbSS, TGF- $\beta 1$ levels above the median (TGF- $\beta 1 \geq 72.29)$ were associated with increased RBC $(p=0.0208)$ (Figure 3(a)), hemoglobin $(p=0.0192)$ (Figure 3(b)), hematocrit $(p=0.0080)$ (Figure 3(c)), monocytes $(p=0.0401) \quad$ (Figure $3(\mathrm{~d}))$, platelets $(p<0.0001)$ (Figure 3(e)), and TIMP-1 $(p<0.0001)$ (Figure 3(f)), as well as decreased albumin $(p=0.0444)$ (Figure $3(\mathrm{~g})$ ). Among individuals with HbSC, TGF- $\beta 1$ levels above the median (TGF- $\beta 1 \geq 47.80)$ were associated with increased leukocytes $(p=0.0049) \quad$ (Figure $4(\mathrm{a}))$, eosinophils $(p=0.0207)$ (Figure 4(b)), lymphocytes $(p=0.0002)$ (Figure 4(c)), monocytes $(p=0.0062)$ (Figure $4(\mathrm{~d}))$, platelets $(p=0.0067)$ (Figure 4(e)), ferritin $(p=0.0125)$ (Figure 4(f)), TIMP-1 $(p=0.0065)$ (Figure 4(g)), MMP-9 $(p=0.0191)$ (Figure 4(h)), triglycerides $(p=0.0102)$ (Figure 5(a)), and VLDL-C $(p=0.0454)$ (Figure 5(b)), as well as decreased HDL-C levels $(p=0.0425)$ (Figure 5(c)).

\section{Discussion}

Despite the fact that several laboratory and genetic biomarkers have been associated with subphenotypes of SCD, in which individuals present a greater propensity of clinical events, the search for prognostic biomarkers of SCD remains challenging $[5,7,24]$.

Our analyses showed that HbSS individuals presented increases in biomarkers of hemolysis, leukocytosis, and inflammation, together with decreased levels of HDL-C, in comparison to $\mathrm{HbSC}$ and $\mathrm{HC}$ individuals. These findings are consistent with previous reports describing HbSS as the most severe form of SCD [25-27]. As individuals with HbSS present more intense hemolysis, anemia tends to be more severe, and hemolytic complications occur more frequently $[24,26]$.

Herein, higher levels of TGF- $\beta 1$ were found in individuals with HbSS than in those with HbSC and HC. Despite the fact that our case series consisted of pediatric individuals, our results were nonetheless similar to a previous report in steady-state adults with HbSS, HbSC, and HC [17]. The elevated TGF- $\beta$ levels found in HbSS may be associated with endothelial remodeling $[12,28]$ since the endothelium controls the release of relaxing and contracting factors that regulate localized vascular tone. Vasculopathy, in addition to 


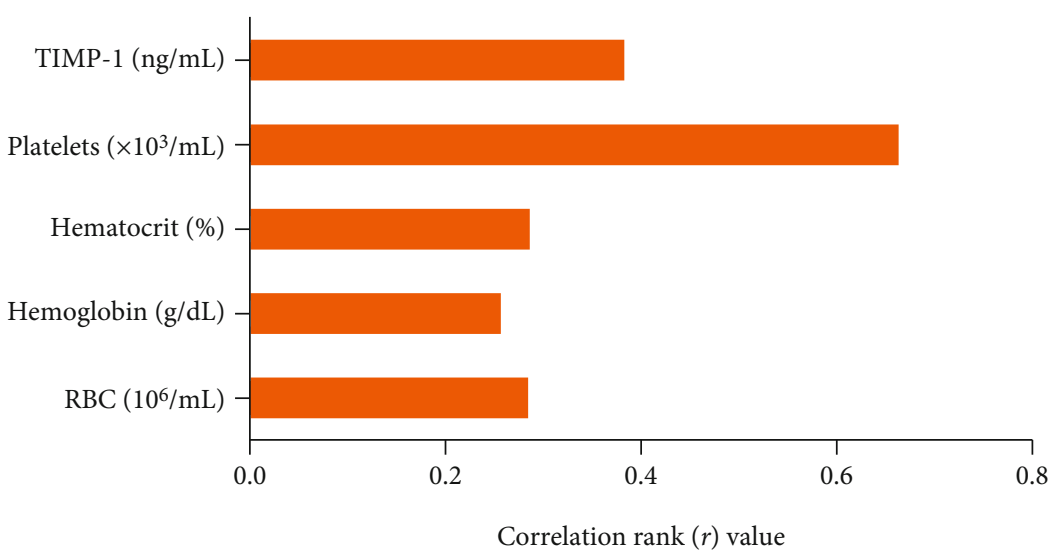

(a)

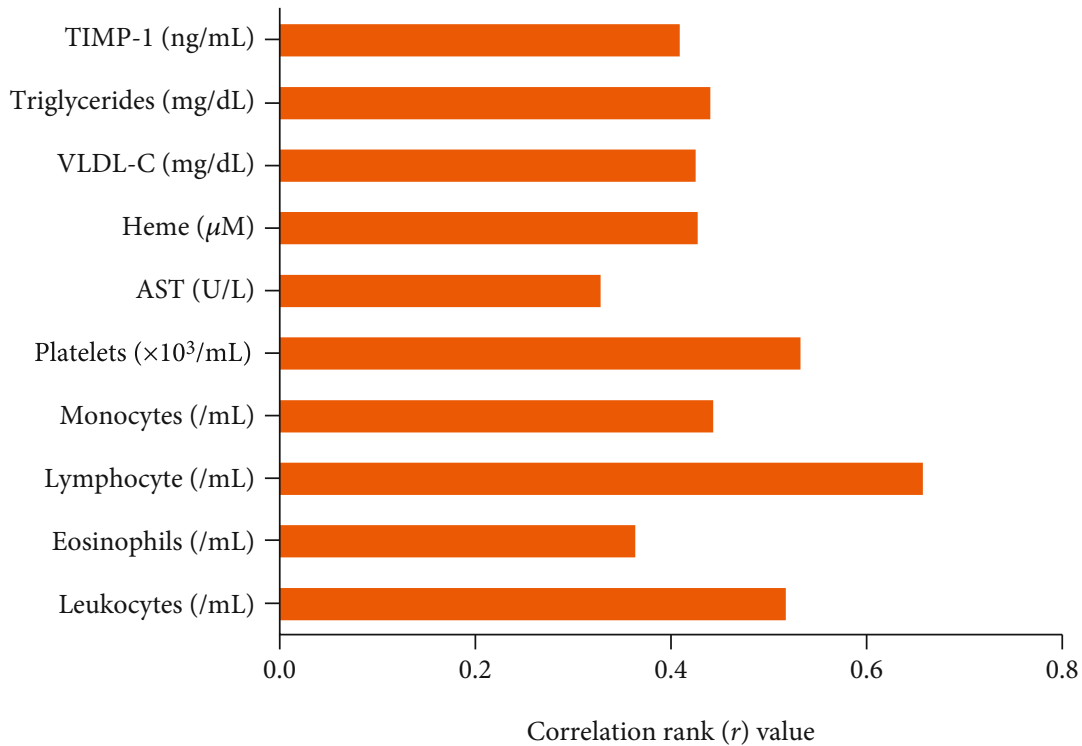

(b)

Figure 2: Correlations between TGF- $\beta 1$ levels and laboratory biomarkers in individuals with HbSS and HbSC. (a) Positive correlations between TGF- $\beta 1$ levels and RBC, Hb, Ht, platelet, and TIMP-1 in individuals with HbSS. (b) Positive correlations between TGF- $\beta 1$ levels and leukocytes, eosinophils, lymphocytes, monocytes, platelets, AST, VLDL-C, triglycerides, heme, and TIMP-1 in HbSC individuals.

endothelial dysfunction, are the main chronic events described in SCD and are also involved in the pathogenesis of stroke, renal disease, and pulmonary hypertension [24]. Thus, it is possible that TGF- $\beta$ could be directly involved in the modulation of vasculopathy in SCD individuals. In addition, elevated TIMP-1 levels were detected in individuals with $\mathrm{HbSS}$ and HbSC who presented TGF- $\beta 1$ levels above the median. TIMP-1, a protein that modulates cell growth, apoptosis, and angiogenesis, is known to inhibit the catalytic activity of MMP-9 [19]. As TGF- $\beta 1$ stimulates the expression of MMP-2, MMP-9, and TIMP-1, increases in TIMP-1 levels driven by TGF- $\beta 1$ may positively impact angiogenesis [29]. Our results also show that individuals with HbSC who presented TGF- $\beta 1$ levels above the median value also exhibited higher levels of MMP-9, which reinforces the role of TGF- $\beta$ in angiogenesis, vasculopathy, and endothelial dysfunction.

The individuals with HbSS and HbSC who presented with TGF- $\beta 1$ levels above the median also exhibited higher platelet counts than those below the median. This finding is supported by previous studies reporting that more TGF- $\beta$ is produced by platelets than by other cells types; moreover, after activation, platelets rapidly release TGF- $\beta[21,30,31]$. Platelet counts were previously shown to be correlated with TGF- $\beta$ levels in both HbSS and HbSC individuals [17], which corroborate the present correlation analysis.

The positive correlation demonstrated herein between TGF- $\beta 1$ and levels of heme and AST in individuals with HbSC provides evidence of the participation of TGF- $\beta 1$ in hemolysis. Interestingly, genes involved in the TGF- $\beta /$ BMP signaling pathway were previously associated with the clinical manifestations of a hemolytic subphenotype, such as leg ulcers [12].

Our association analysis revealed that individuals with HbSC and TGF- $\beta 1$ levels above the median presented increased leukocyte counts. In addition, our correlation analysis found a positively correlation between TGF- $\beta 1$ levels and leukocyte, eosinophil, lymphocyte, and monocyte counts, indicating the presence of an inflammatory response. 


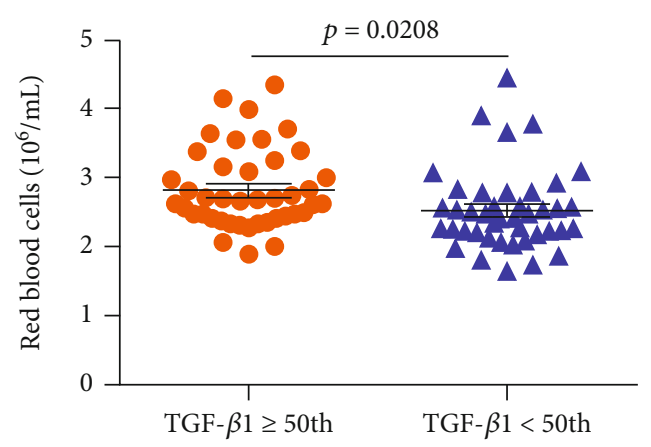

(a)

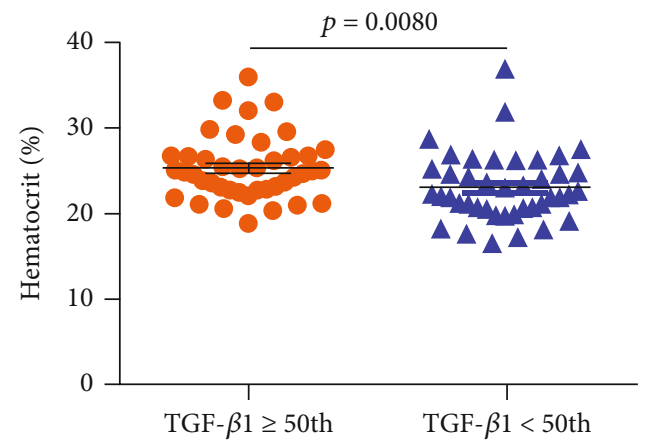

(c)

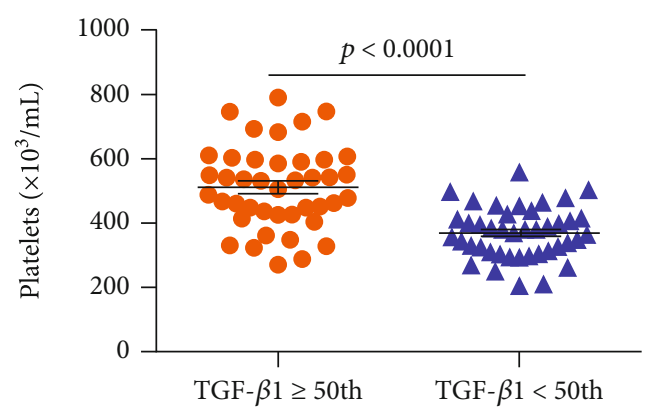

(e)

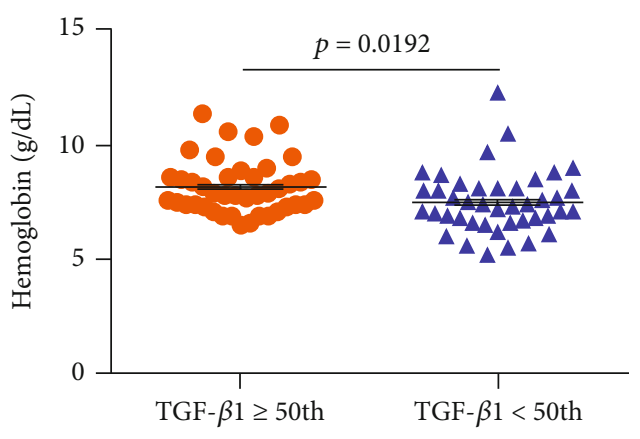

(b)

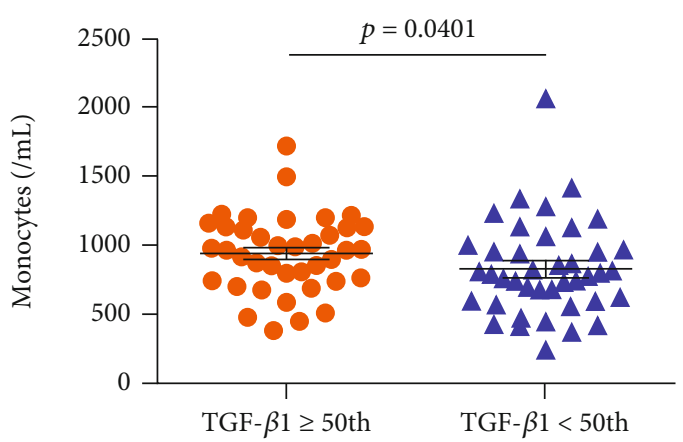

(d)

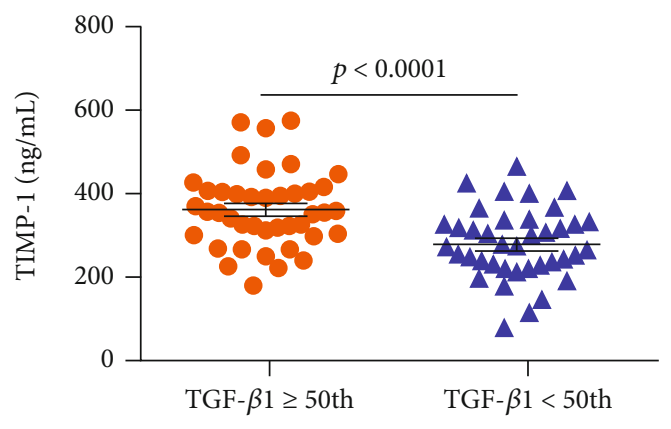

(f)

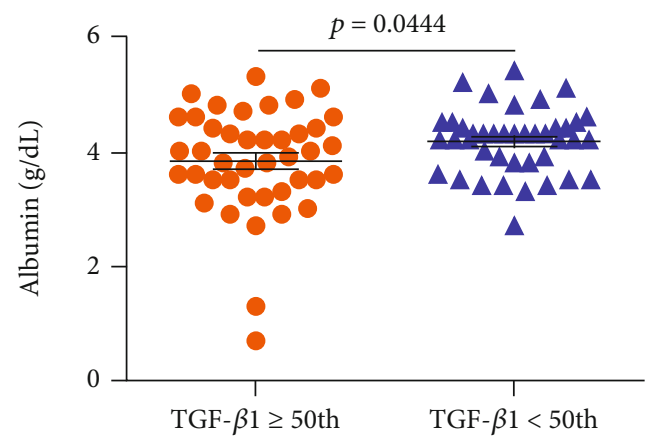

(g)

FIgURE 3: Associations between TGF- $\beta 1$ levels and laboratory biomarkers as well as the glycoprotein TIMP-1 in individuals with HbSS. Individuals with TGF- $\beta 1$ levels above the median (TGF- $\beta 1 \geq 72.29$ ) exhibited increased (a) RBC counts, (b) hemoglobin levels, (c) hematocrit, (d) monocyte counts, (e) platelet counts, and (f) TIMP-1 levels, as well as (g) decreased albumin levels. All $p$ values were obtained by the Mann-Whitney $U$ test, except for TIMP-1 and platelet counts, for which the independent $t$-test was used.

Similarly, a previous study reported a correlation between TGF- $\beta$ levels and total leukocyte counts in individuals with $\mathrm{HbS} \beta$-thalassemia [17]. TGF- $\beta$ is involved in neutrophil and monocyte chemotaxis, which influences leukocyte recruitment to inflammatory sites [32]. In the inflammatory state seen in SCD individuals, markedly high expressions of 


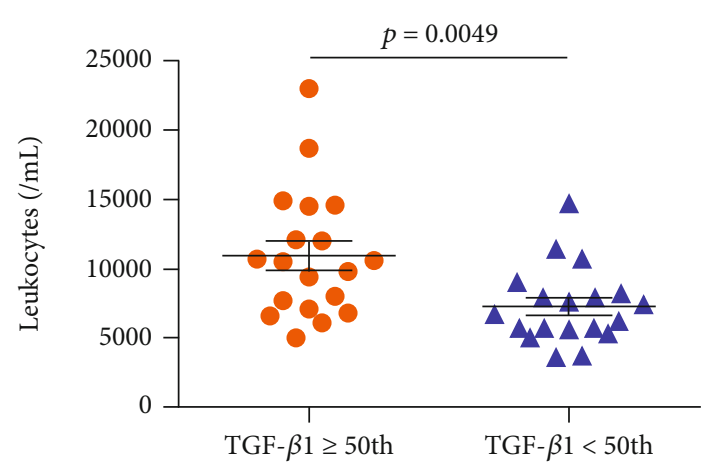

(a)

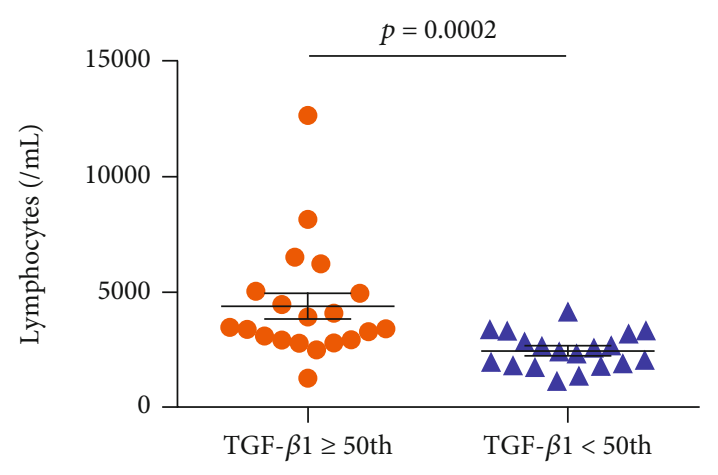

(c)

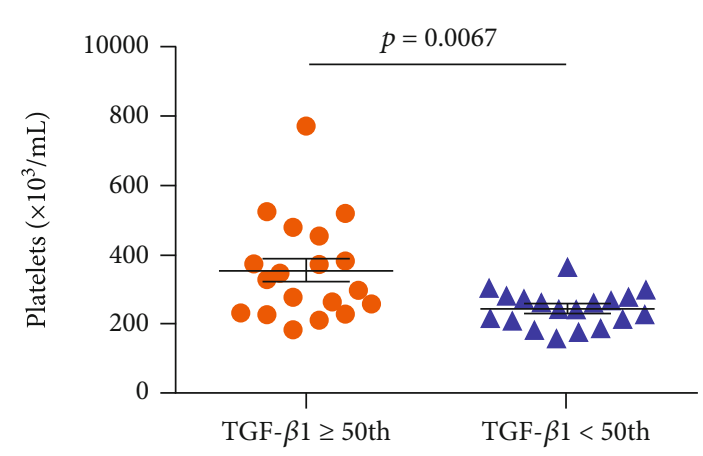

(e)

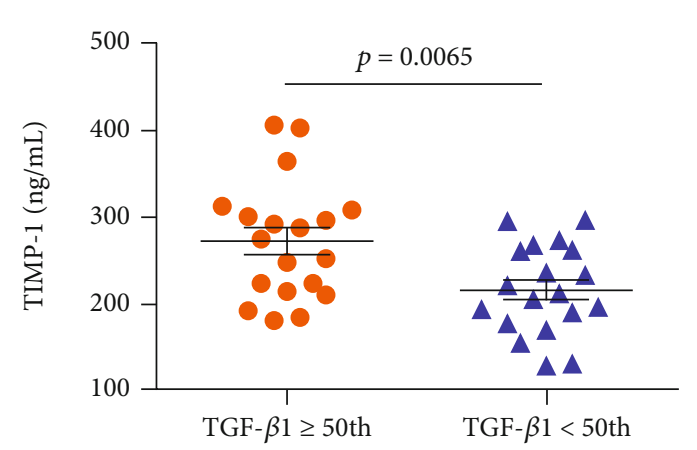

(g)

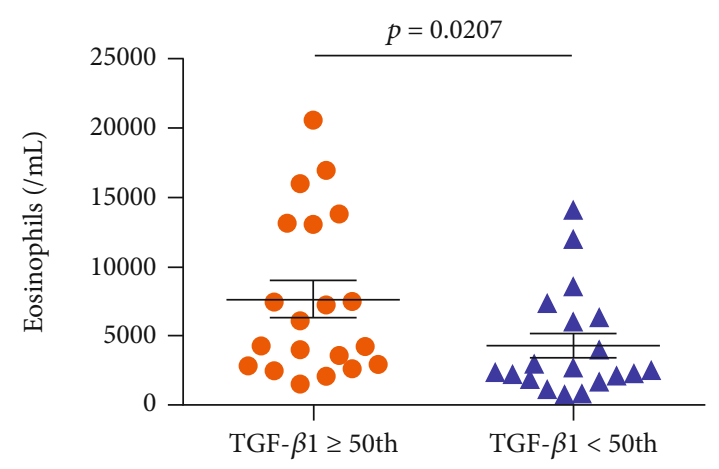

(b)

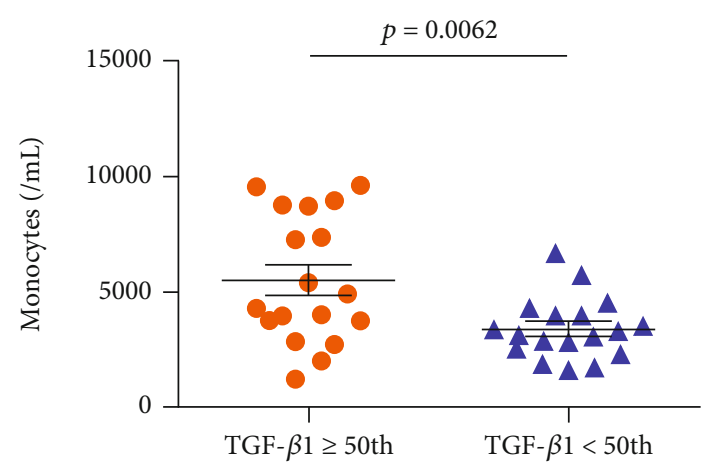

(d)

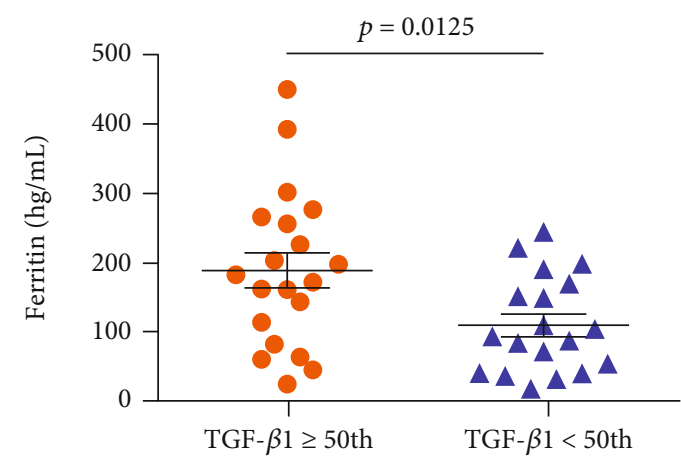

(f)

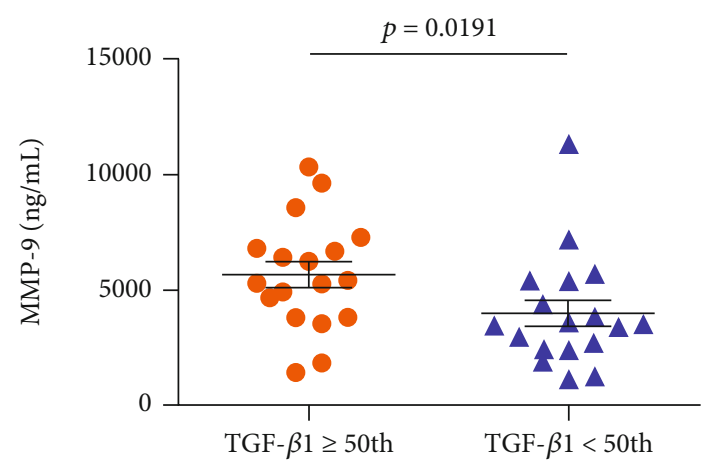

(h)

FIGURE 4: Associations between TGF- $\beta 1$ levels and laboratory biomarkers as well as the glycoprotein TIMP-1 and matrixin MMP-9 in individuals with HbSC. Individuals with TGF- $\beta 1$ levels above the median (TGF- $\beta 1 \geq 47.80$ ) exhibited increased (a) leukocytes, (b) eosinophils, (c) lymphocytes, (d) monocytes, and (e) platelets, as well as higher levels of (f) ferritin, (g) TIMP-1, and (h) MMP-9. All $p$ values were obtained by the Mann-Whitney $U$ test, except for leukocytes, monocytes, ferritin, and TIMP-1, for which the independent $t$-test was used. 


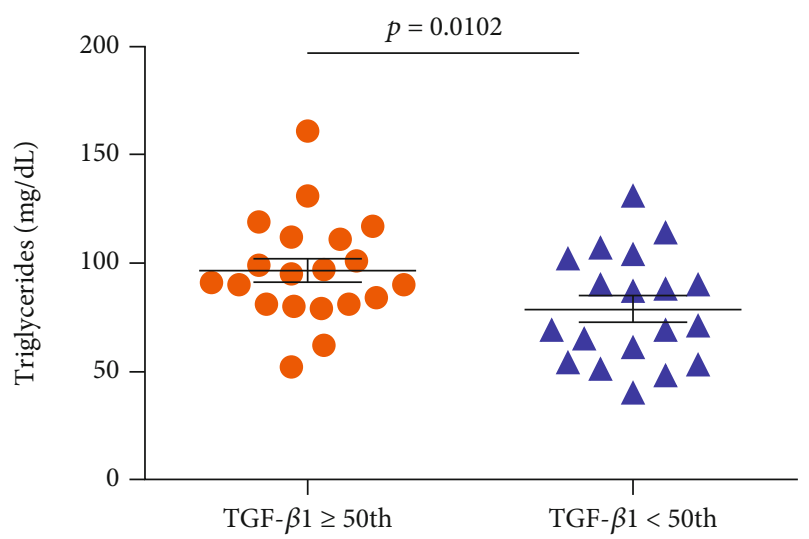

(a)

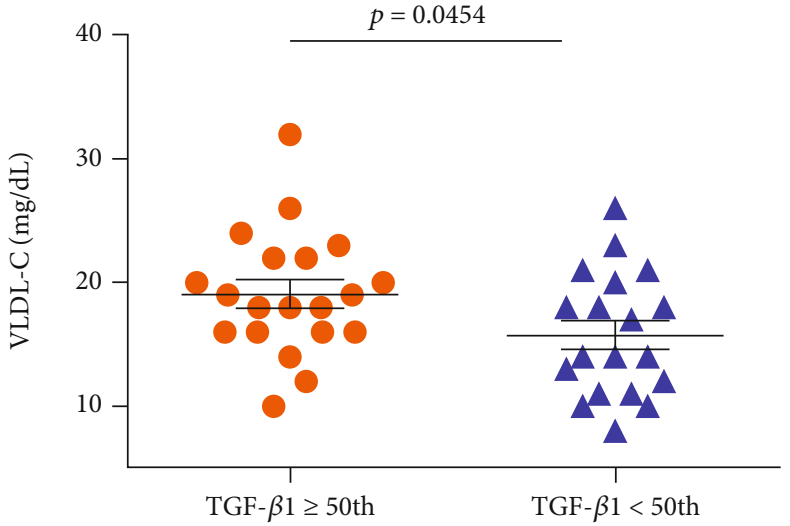

(b)

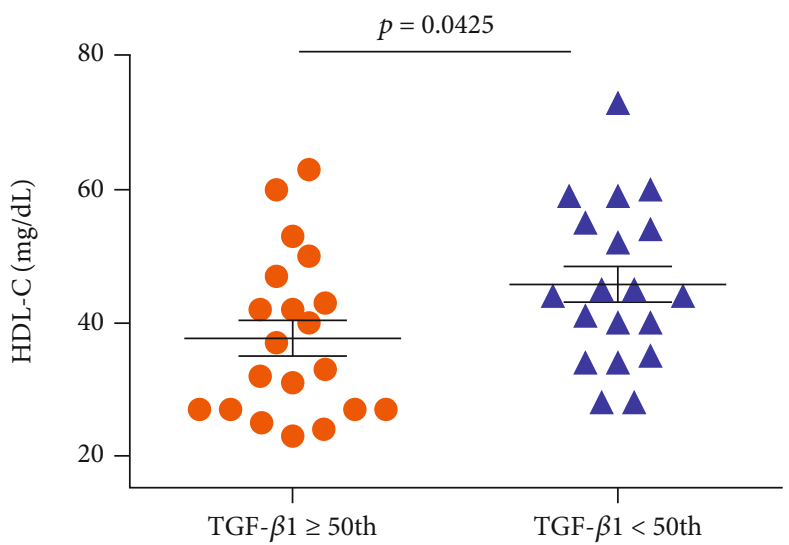

(c)

FIGURE 5: Associations between TGF- $\beta 1$ levels and lipid biomarkers in individuals with HbSC. Individuals with TGF- $\beta 1$ levels above the median (TGF- $\beta 1 \geq 47.80$ ) exhibited (a) increased triglycerides, (b) increased VLDL-C, and decreased (c) HDL-C levels (all $p$ values were obtained by the independent $t$-test).

adhesion molecules, as well as the production of chemotactic factors and inflammatory cytokines, all promote leukocyte recruitment $[2,8,33]$.

Regarding the lipid profile, HbSC individuals with TGF$\beta 1$ levels above the median presented decreased HDL-C. In addition, TGF- $\beta 1$ levels were also found to be positively correlated with VLDL-C and triglycerides in these individuals. HDL-C exerts important anti-inflammatory activity in vascular diseases [34-36] In SCD, decreased HDL-C levels have been associated with an inflammatory state [7, 34]. Triglycerides and VLDL-C have been shown to induce inflammatory events in atherosclerosis, supporting the notion that these molecules participate in proinflammatory activity [37].

Our findings suggest that TGF- $\beta 1$ levels bear relations to vasculopathy, endothelial dysfunction, hemolysis, and inflammation in individuals with SCD. Collectively, our results highlight the relevance of investigating novel biomarkers of disease severity in the clinical management of individuals with HbSS and HbSC. To the best of our knowledge, the present study was the first to investigate associations and correlations between TGF- $\beta 1$ levels and hematological and biochemical parameters in a pediatric population with $\mathrm{HbSS}$ and $\mathrm{HbSC}$. We emphasize that the identification of markers indicative of a worse prognosis of SCD in children can greatly aid in the improved clinical management of patients.

\section{Conclusion}

The results presented herein suggest that pediatric patients with HbSS present higher levels of TGF- $\beta 1$ than those with HbSC or healthy individuals. The fact that TGF- $\beta 1$ was associated with TIMP-1 in both genotypes indicates that these molecules may play an important role in vascular remodeling and vasculopathy through extracellular matrix deposition. We suggest that since TGF- $\beta$ was found to be associated with hemolysis, leukocytes, platelets, and lipid metabolism, this provides evidence that this immunomarker likely modulates the inflammatory response in SCD.

In summary, the full spectrum of biological effects provoked by TGF- $\beta$ in SCD warrants further investigation, as evidence points to the involvement of this molecule in the pathogenesis of vascular disease.

\section{Data Availability}

All relevant data used to support the findings of this study are included within the article. 


\section{Disclosure}

The sponsors of this study, who played no role in gathering, analyzing, or interpreting the data presented herein, are public organizations whose role is to support science in general.

\section{Conflicts of Interest}

The authors declare that there is no conflict of interest regarding the publication of this paper.

\section{Authors' Contributions}

Rayra P. Santiago and Magda O. S. Carvalho contributed equally to this study.

\section{Acknowledgments}

We would like to thank all the SCA individuals who agreed to participate in our research protocol. We also thank the staff of the Bahia State Hematology and Hemotherapy Foundation (HEMOBA) for their assistance with sample collection and for caring for SCD individuals. The authors are grateful to Andris K. Walter for the English revision and manuscript copyediting services. This study was financed in part by the Coordenação de Aperfeiçoamento de Pessoal de Nível Superior-Brasil (CAPES), Finance Code 001 (RPS and SCMAY). Our work was also supported by the Fundação de Amparo à Pesquisa do Estado da Bahia (FAPESB SUS0034/2013 and 8133/2014) through a grant to MSG.

\section{References}

[1] D. C. Rees, S. L. Thein, A. Osei et al., "The clinical significance of $\mathrm{K}-\mathrm{Cl}$ cotransport activity in red cells of patients with $\mathrm{HbSC}$ disease," Haematologica, vol. 100, no. 5, pp. 595-600, 2015.

[2] G. J. Kato, F. B. Piel, C. D. Reid et al., "Sickle cell disease," Nature Reviews Disease Primers, vol. 4, no. 1, article 18010, 2018.

[3] R. P. Santiago, C. Vieira, C. S. A. Adanho et al., "Laboratory and genetic biomarkers associated with cerebral blood flow velocity in hemoglobin SC disease," Disease Markers, vol. 2017, Article ID 6359871, 11 pages, 2017.

[4] C. C. da Guarda, S. C. M. A. Yahouédéhou, R. P. Santiago et al., "Sickle cell disease: a distinction of two most frequent genotypes (HbSS and HbSC)," PLoS One, vol. 15, no. 1, pp. e0228399-e0228399, 2020.

[5] G. J. Kato, M. T. Gladwin, and M. H. Steinberg, "Deconstructing sickle cell disease: reappraisal of the role of hemolysis in the development of clinical subphenotypes," Blood Reviews, vol. 21, no. 1, pp. 37-47, 2007.

[6] M. H. Steinberg, "Genetic etiologies for phenotypic diversity in sickle cell anemia," ScientificWorldJournal, vol. 9, pp. 46-67, 2009.

[7] M. M. Aleluia, C. C. da Guarda, R. P. Santiago et al., "Association of classical markers and establishment of the dyslipidemic sub-phenotype of sickle cell anemia," Lipids in Health and Disease, vol. 16, no. 1, p. 74, 2017.

[8] C. C. D. Guarda, R. P. Santiago, L. M. Fiuza et al., "Hememediated cell activation: the inflammatory puzzle of sickle cell anemia," Expert Review of Hematology, vol. 10, no. 6, pp. $533-$ 541, 2017.

[9] C. Lanaro, C. F. Franco-Penteado, D. M. Albuqueque, S. T. O. Saad, N. Conran, and F. F. Costa, "Altered levels of cytokines and inflammatory mediators in plasma and leukocytes of sickle cell anemia patients and effects of hydroxyurea therapy," Journal of Leukocyte Biology, vol. 85, no. 2, pp. 235-242, 2009.

[10] W. Vilas-Boas, B. A. Veloso Cerqueira, C. V. B. Figueiredo et al., "Association of homocysteine and inflammatoryrelated molecules in sickle cell anemia," Hematology, vol. 21, no. 2, pp. 126-131, 2015.

[11] M. O. S. Carvalho, T. Araujo-Santos, J. H. O. Reis et al., "Inflammatory mediators in sickle cell anaemia highlight the difference between steady state and crisis in paediatric patients," British Journal of Haematology, vol. 182, no. 6, pp. 933-936, 2018.

[12] V. G. Nolan, A. Adewoye, C. Baldwin et al., "Sickle cell leg ulcers: associations with haemolysis and SNPs in Klotho, TEK and genes of the TGF-beta/BMP pathway," British Journal of Haematology, vol. 133, no. 5, pp. 570-578, 2006.

[13] L. Attisano and J. L. Wrana, "Signal transduction by the TGFbeta superfamily," Science, vol. 296, no. 5573, pp. 1646-1647, 2002.

[14] K. Miyazono, K. Kusanagi, and H. Inoue, "Divergence and convergence of TGF-beta/BMP signaling," Journal of Cellular Physiology, vol. 187, no. 3, pp. 265-276, 2001.

[15] F. N. Ziyadeh, "Mediators of diabetic renal disease: the case for TGF-? as the major mediator," J Am Soc Nephrol, vol. 15, no. 90010, pp. 55S-557, 2004.

[16] A. Moustakas, K. Pardali, A. Gaal, and C. H. Heldin, "Mechanisms of TGF-beta signaling in regulation of cell growth and differentiation," Immunology Letters, vol. 82, no. 1-2, pp. 8591, 2002.

[17] L. . S. Torres, J. V. Okumura, D. G. H. . Silva et al., "Plasma levels of TGF- $\beta 1$ in homeostasis of the inflammation in sickle cell disease," Cytokine, vol. 80, pp. 18-25, 2016.

[18] H.-L. Hsieh, H. H. Wang, W. B. Wu, P. J. Chu, and C. M. Yang, "Transforming growth factor- $\beta 1$ induces matrix metalloproteinase- 9 and cell migration in astrocytes: roles of ROS-dependent ERK- and JNK-NF- $\kappa$ B pathways," Journal of Neuroinflammation, vol. 7, no. 1, pp. 88-88, 2010.

[19] H.-J. Kwak, M. J. Park, H. Cho et al., “Transforming growth factor- $\beta 1$ induces tissue inhibitor of metalloproteinase-1 expression via activation of extracellular signal-regulated kinase and Sp1 in human fibrosarcoma cells," Molecular Cancer Research, vol. 4, no. 3, pp. 209-220, 2006.

[20] R. Khokha, A. Murthy, and A. Weiss, "Metalloproteinases and their natural inhibitors in inflammation and immunity," Nature Reviews. Immunology, vol. 13, no. 9, pp. 649-665, 2013.

[21] P. Seizer and A. E. May, "Platelets and matrix metalloproteinases," Thrombosis and Haemostasis, vol. 110, no. 5, pp. 903909, 2013.

[22] S.-A. Park, M. J. Kim, S. Y. Park et al., “TIMP-1 mediates TGF- $\beta$ dependent crosstalk between hepatic stellate and cancer cells via FAK signaling," Scientific Reports, vol. 5, no. 1, p. 16492, 2015.

[23] S.-K. Leivonen, K. Lazaridis, J. Decock, A. Chantry, D. R. Edwards, and V. M. Kähäri, "TGF- $\beta$-elicited induction of tissue inhibitor of metalloproteinases (TIMP)-3 expression in fibroblasts involves complex interplay between Smad3, p38 $\alpha$, and ERK1/2," PLoS One, vol. 8, no. 2, pp. e57474-e57474, 2013. 
[24] D. C. Rees and J. S. Gibson, "Biomarkers in sickle cell disease," British Journal of Haematology, vol. 156, no. 4, pp. 433-445, 2012.

[25] M. H. Steinberg and G. P. Rodgers, "Pathophysiology of sickle cell disease: role of cellular and genetic modifiers," Seminars in Hematology, vol. 38, no. 4, pp. 299-306, 2001.

[26] R. L. Nagel, M. E. Fabry, and M. H. Steinberg, "The paradox of hemoglobin SC disease," Blood Reviews, vol. 17, no. 3, pp. 167178, 2003.

[27] F. Lionnet, N. Hammoudi, K. S. Stojanovic et al., "Hemoglobin sickle cell disease complications: a clinical study of 179 cases," Haematologica, vol. 97, no. 8, pp. 1136-1141, 2012.

[28] J. E. Brittain and L. V. Parise, "Cytokines and plasma factors in sickle cell disease," Current Opinion in Hematology, vol. 14, no. 5, pp. 438-443, 2007.

[29] I. Pantsulaia, S. Trofimov, E. Kobyliansky, and G. Livshits, "Heritability of circulating growth factors involved in the angiogenesis in healthy human population," Cytokine, vol. 27, no. 6, pp. 152-158, 2004.

[30] R. K. Assoian, A. Komoriya, C. A. Meyers, D. M. Miller, and M. B. Sporn, "Transforming growth factor-beta in human platelets. Identification of a major storage site, purification, and characterization," The Journal of Biological Chemistry, vol. 258, no. 11, pp. 7155-7160, 1983.

[31] J. Ahamed, N. Burg, K. Yoshinaga, C. A. Janczak, D. B. Rifkin, and B. S. Coller, "In vitro and in vivo evidence for shearinduced activation of latent transforming growth factorbeta1," Blood, vol. 112, no. 9, pp. 3650-3660, 2008.

[32] N. G. Frangogiannis, "The role of transforming growth factor (TGF)- $\beta$ in the infarcted myocardium," Journal of Thoracic Disease, vol. 9, Supplement 1, pp. S52-S63, 2017.

[33] P. Sundd, M. T. Gladwin, and E. M. Novelli, "Pathophysiology of sickle cell disease," Annual Review of Pathology: Mechanisms of Disease, vol. 14, no. 1, pp. 263-292, 2019.

[34] M. O. Seixas, L. C. Rocha, M. B. Carvalho et al., "Levels of high-density lipoprotein cholesterol (HDL-C) among children with steady-state sickle cell disease," Lipids in Health and Disease, vol. 9, no. 1, p. 91, 2010.

[35] R. K. Ephraim, P. Adu, E. Ake et al., "Normal non-HDL cholesterol, low total cholesterol, and HDL cholesterol levels in sickle cell disease patients in the steady state: a case-control study of Tema Metropolis," Journal of Lipids, vol. 2016, Article ID 7650530, 5 pages, 2016.

[36] C. C. da Guarda, S. C. M. A. Yahouédéhou, R. P. Santiago et al., "Investigation of lipid profile and clinical manifestations in SCA children," Disease Markers, vol. 2020, Article ID 8842362, 10 pages, 2020.

[37] F. K. Welty, "How do elevated triglycerides and low HDLcholesterol affect inflammation and atherothrombosis?," Current Cardiology Reports, vol. 15, no. 9, pp. 400-400, 2013. 